\title{
Análisis Didáctico a un Proceso de Instrucción del Método de Integración por Partes*
}

\section{Teaching Analysis to Process Integration Method Instruction by Parties}

\author{
Enrique Mateus Nieves ${ }^{* *}$
}

\begin{abstract}
Resumen
En este artículo se presenta un análisis de la estructura y funcionamiento de una secuencia de clases de matemáticas, con estudiantes colombianos de segundo año de la licenciatura en matemáticas, donde se explica el método de integración por partes. Para ello, se utiliza el modelo de análisis didáctico propuesto por el Enfoque Ontosemiótico de la Cognición e Instrucción Matemática. El análisis didáctico realizado permite concluir que la secuencia de clases analizada se puede considerar como una degeneración mecanicista de la clase formal. Dado que en el desarrollo de la misma se utilizan en forma parcial características propias del paradigma formalmecanicista. Por otra parte, se observa que la estructura y funcionamiento de la clase analizada no tiene en cuenta la complejidad ontosemiótica ${ }^{* * * *}$ de la integral, lo cual es una de las causas de que se produzcan determinadas dificultades de aprendizaje en los alumnos.
\end{abstract}

Palabras-clave: Análisis Didáctico. Integración. Integración por Partes. Idoneidad Didáctica.

\begin{abstract}
This article presents an analysis of the structure and functioning of a sequence of math classes, with Colombian sophomore bachelor's degree in mathematics, where the method of integration by parts explained is presented. In order to do so, the model of analysis proposed by the Focus Ontosemiotic of Cognition and Instruction Mathematics was used. The didactic analysis leads to the conclusion that the sequence analyzed classes can be considered as a mechanistic degeneration of the formal class. Since the development of the same are used partially formal characteristics mechanistic paradigms. Moreover, it is observed that the structure and operation of the analyzed classes ignores the complexity of integrated ontosemiotic, which is one of the reasons why certain learning difficulties occur in students.
\end{abstract}

Keywords: Didactic Analysis.Integral, Integration by Parts. Suitability Teaching.

\footnotetext{
* Documento resultado del trabajo de investigación doctoral orientada por el Dr. Carlos Vasco Uribe, con el apoyo internacional del Dr. Vicent Font Moll, Departament de Didàctica de les Ciències Experimentals i la Matemàtica, Facultat de Formació del Professorat, Universitat de Barcelona, en 2014

Estudiante Doctorado Interinstitucional en Educación, énfasis Educación Matemática por la Universidad Distrital "Francisco José de Caldas" (DIE-UD), Bogotá, Colombia. Dirección postal: Carrera 30 \# 64-06, piso 3 , Bogotá D. C., Colombia; E-mail: jeman124@gmail.com.

*** Entendiendo por Complejidad Ontosemiótica la técnica de análisis reconocida como un recurso útil para la investigación en Didáctica de las Matemáticas (GODINO, 2002; Godino et al., 2010) que permite hacer un análisis de tipo microscópico con el propósito de identificar significados puestos en juego en una activada matemática puntual, y, de manera más general, permite describir la estructura semiótica de una organización matemática compleja implementada en un proceso de estudio particular.
} 


\section{Introducción}

Partimos de la base de que los objetos matemáticos tienen una naturaleza compleja que está distribuida en instituciones diferentes, en momentos históricos diferentes, en libros de textos diferentes etc. Nos interesa conocer cómo ciertos aspectos de esta complejidad del objeto matemático se ponen de manifiesto en la práctica del profesor de matemáticas cuando lo está enseñando. Partimos del siguiente supuesto: la manera cómo se tiene en cuenta la complejidad del objeto matemático a enseñar (en términos definiciones, proposiciones, propiedades, procesos matemáticos etc.) y cómo ésta se gestiona en el proceso de instrucción, es un aspecto clave para entender las dificultades de aprendizaje de los alumnos.

Son diversos los enfoques teóricos que se han interesado por analizar la práctica docente del profesor de matemáticas, entre otros: 1) los trabajos de Rowland, Huckstep, y Thwaites (2005), su propuesta de cuatro categorías de conocimiento (foundation, transformation, connection y contingency) que caracterizan el conocimiento del profesor activado durante la instrucción; 2) las aportaciones derivadas de la metodología Lesson Study (FERNÁNDEZ; YOSHIDA, 2004) que incluyen un modelo de análisis colaborativo por parte del profesorado para planificar, implementar, observar, y reflexionar sobre las clases de matemáticas; 3) Las aportaciones de Davis y Renert (2013) en el marco de su propuesta Concept Study, que combina elementos de dos enfoques relevantes en la investigación en educación matemática: concept analysis y lesson study; 4) las investigaciones sobre el desarrollo y evaluación de la competencia denominada mirar con sentido el pensamiento matemático de los estudiantes (MASON, 2002).

Dicha competencia permite al profesor de matemáticas ver las situaciones de enseñanza aprendizaje de las matemáticas de una manera profesional, que lo diferencia de la manera de mirar de alguien que no es profesor de matemáticas; 5) el modelo de análisis didáctico propuesto por el Enfoque Ontosemiótico de la Instrucción y la Cognición Matemática (EOS); este enfoque propone cinco niveles para el análisis didáctico de procesos de instrucción propios y ajenos, cada uno de ellos con sus respectivas herramientas (GODINO; CONTRERAS; FONT, 2006; FONT, PLANAS y GODINO, 2010; POCHULU; FONT; RODRÍGUEZ, 2015): (1) Análisis de los tipos de problemas y sistemas de prácticas; (2) Análisis de las configuraciones de objetos y procesos matemáticos; (3) Análisis de evoluciones cognitivas, trayectorias e interacciones didácticas, (4) Identificación del sistema de normas y metanormas y (5) Valoración de la idoneidad didáctica del proceso de instrucción para identificar y potenciar buenas prácticas. 
El objetivo de este trabajo es realizar un análisis minucioso del proceso de instrucción realizado en una secuencia de clases, utilizando el modelo de análisis didáctico propuesto por el EOS. Se trata de determinar la estructura y el funcionamiento de una secuencia de clases de matemáticas, con estudiantes colombianos de segundo año de la licenciatura en matemáticas, donde se explica el método de integración por partes. Dicho análisis permite describir (¿qué ha ocurrido aquí?), explicar (¿por qué ha ocurrido?) y valorar (¿qué se podría mejorar?) el proceso de instrucción realizado. El principal resultado es una valoración fundamentada de la idoneidad didáctica del proceso de instrucción implementado y una explicación de las dificultades de aprendizaje de los alumnos.

Este artículo está organizado en nueve secciones, la primera de los cuales es esta introducción en la que se expone el objetivo de la investigación. En la segunda sección, se explica el contexto donde se realizó la secuencia de clases que se ha analizado. En la sección 3, explicamos las herramientas de los cinco niveles de análisis didáctico propuestos por el EOS y en las secciones $4-7$, comentamos cómo se han usado para el análisis de la secuencia de clases, poniendo énfasis en la configuración didáctica $n^{\circ} 2$ (CD2) (en el anexo 1 se muestra su trascripción aunque, por razones de espacio, se omiten algunas líneas). En la sección 7 realizamos la valoración de la idoneidad didáctica basándonos en el estudio de los aspectos descriptivos y explicativos plasmados en las secciones 4 - 6. A continuación, en la sección 8 , explicamos las dificultades que tienen los alumnos en la resolución de problemas de integración por partes a partir del análisis realizado. El artículo finaliza con una presentación de consideraciones finales.

\section{Contextualización de la clase analizada}

Las clases consideradas, que tienen una duración aproximada de una hora, fueron 12. Algunas de ellas se implementaron según el horario asignado en sesiones de hora y media de clase. Se impartieron en la asignatura de Cálculo Integral, durante el tercer semestre de la licenciatura en matemáticas de una universidad colombiana, con 20 alumnos de 21 a 23 años de edad. La universidad es de tipo pública estatal. El profesor tenía una antigüedad en la docencia de cinco años, una formación en matemática pura y una especialización en docencia de la matemática universitaria.

Para el análisis, hemos subdividido las 12 sesiones de clase en 820 unidades de análisis, que hemos agrupado en episodios de aula que denominamos configuraciones didácticas (GODINO; CONTRERAS; FONT, 2006), de acuerdo con el marco teórico y metodológico 
adoptado para el trabajo (EOS). Cada una de las configuraciones didácticas (CD) muestra las interacciones en torno a una tarea matemática y finaliza cuando se inicia otra. Tal como se señala en Font, Bolite y Acevedo (2010); Pochulu y Font (2011), aunque el criterio básico para determinar una $\mathrm{CD}$ es la realización de una tarea, la agrupación de las líneas de la transcripción en configuraciones didácticas es flexible y queda a criterio del investigador.

\section{Niveles de análisis didáctico propuesto por el EOS}

Para realizar el análisis de la transcripción de las clases, optamos por el modelo de análisis didáctico propuesto por el EOS (FONT; PLANAS; GODINO, 2010), que considera los siguientes cinco niveles o tipos de análisis:

1) Identificación de prácticas matemáticas

2) Elaboración de las configuraciones didácticas y epistémicas de objetos y procesos matemáticos

3) Análisis de las trayectorias e interacciones didácticas

4) Identificación del sistema de normas y metanormas

5) Valoración de la idoneidad didáctica del proceso de instrucción

El primer nivel de análisis explora las prácticas matemáticas de un proceso de instrucción matemático. Se puede entender como la narración que haría un profesor para explicar a otro lo que ha sucedido desde el punto de vista matemático. El análisis didáctico debe progresar desde la situación problema o tarea básica propuesta y las prácticas matemáticas necesarias para su resolución (nivel 1) a los objetos y procesos matemáticos que posibilitan dichas prácticas. El segundo nivel de análisis se centra en los objetos y procesos matemáticos que intervienen en la realización de las prácticas, así como los que emergen de ellas. Dado que el estudio de las matemáticas tiene lugar usualmente bajo la dirección de un profesor y en interacción con otros estudiantes, se debe progresar hacia el estudio de la interacción. El tercer nivel de análisis didáctico está orientado, sobre todo, a la descripción de los patrones de interacción, a las configuraciones didácticas y su articulación secuencial en trayectorias didácticas (nivel 3); las configuraciones y trayectorias están condicionadas y soportadas por una trama de normas y metanormas que no sólo regulan la dimensión epistémica de los procesos de instrucción (niveles 1 y 2), sino también otras dimensiones de estos procesos (cognitiva, afectiva etc.). El cuarto nivel de análisis estudia dicha trama.

Los cuatro primero niveles de análisis son herramientas para una didáctica descriptivaexplicativa, mientras que el quinto se centra en la valoración de la idoneidad didáctica 
(GODINO et al., 2006). Este último nivel se basa en los cuatro análisis previos y es una síntesis orientada a la identificación de mejoras potenciales del proceso de instrucción en nuevas implementaciones. El análisis de las prácticas, objetos y procesos matemáticos permite describir las matemáticas del proceso de instrucción analizado, mientras que el de las interacciones y de la dimensión normativa permite describir la interacción producida en el proceso de instrucción y las normas que la regulan.

Por último, los criterios de idoneidad implican la incorporación de una racionalidad axiológica en la educación matemática que permita el análisis, la crítica, la justificación de la elección de los medios y de los fines, la justificación del cambio etc. Las herramientas de los cuatro primeros niveles de análisis propuestos en el EOS permiten descomponer una transcripción de una sesión de clases en una trayectoria de configuraciones didácticas y, para cada configuración, estudiar diferentes aspectos. Por ejemplo, la configuración didáctica $\mathrm{n}^{\mathbf{0}} 2$ (CD2), que es la que utilizaremos en este artículo, va de la línea 33 de la transcripción a la 122 (ver Anexo 1, donde por cuestiones de espacio se omiten algunas líneas). Dicha CD2 comienza con una tarea propuesta por el profesor (Calcular la integral $\int x e^{3 x} d x$ ) y termina cuando el profesor propone otra tarea (calcular otra integral).

En esta configuración CD2, la práctica matemática realizada consiste en aprender el método de la integración por partes a partir de su aplicación en un caso particular. Los objetos de la configuración epistémica que se ponen en juego en dicha práctica son, entre otros, antiderivada, integral indefinida, derivada de un producto de funciones etc., mientras que uno de los procesos activados es el tratamiento de expresiones simbólicas. En esta configuración didáctica la intervención del profesor se ha concretado en presentar (institucionalizar) el método de integración por partes a partir de la resolución de una integral particular. El patrón de interacción sirve para tipificar la configuración didáctica como magistral interactiva. Se observan varios conflictos semióticos potenciales causados por las explicaciones ambiguas del profesor y por el uso de simbología imprecisa. Por otra parte, en esta CD2 y en otras, se infieren, por ejemplo, que las normas que regulan la interacción son los de una clase magistral. Los cursos están concebidos en forma expositiva ilustrativa, con tareas y actividades específicas propias de esa modalidad.

\section{Identificación de prácticas matemáticas, objetos y procesos matemáticos}


Durante esta secuencia de las 12 sesiones de clase, el profesor pretende enseñar el método de integración por partes. Los alumnos, sobre todo, realizan la práctica de resolver integrales por este método de integración. En particular, esta es la práctica que se realiza en CD2. Si consideramos los aspectos del conocimiento para la realización y evaluación de la práctica que permiten resolver una situación problema - por ejemplo, calcular una integral vemos el uso de lenguajes verbales y simbólicos. Estos lenguajes son la parte ostensiva de una serie de conceptos, proposiciones y procedimientos que intervienen en la elaboración de argumentos para decidir si las acciones realizadas son satisfactorias. Así, cuando un agente lleva a cabo y evalúa una práctica matemática, activa un conglomerado formado por situaciones problema, lenguajes, conceptos, proposiciones, procedimientos y argumentos. Para la CD2, que es el episodio que analizamos con detalle, dichos elementos se articulan en la siguiente configuración epistémica ${ }^{1}$ (Cuadro 1).

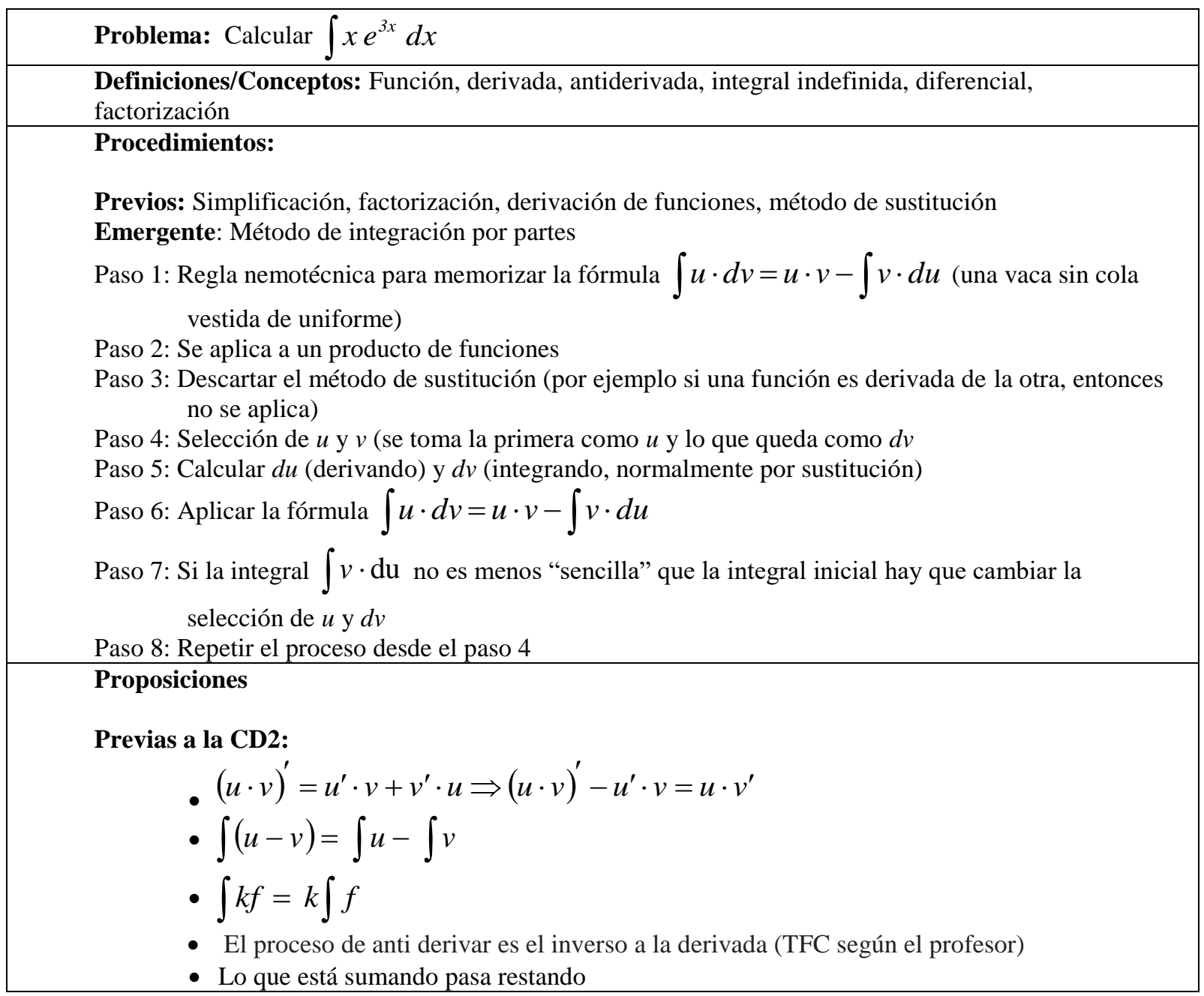

\footnotetext{
${ }^{1}$ Se denominará configuración epistémica al sistema de objetos y funciones semióticas que se establecen entre ellos relativos a la resolución de una situación-problema. Por tanto, se trata de un segmento de la trayectoria de enseñanza como un aspecto de la trayectoria didáctica global, que, una vez organizada por configuraciones epistémicas, puede considerarse como trayectoria epistémica. Cada configuración epistémica, globalmente considerada, desempeña una función específica en el proceso de instrucción.
} 


\begin{tabular}{|c|}
\hline 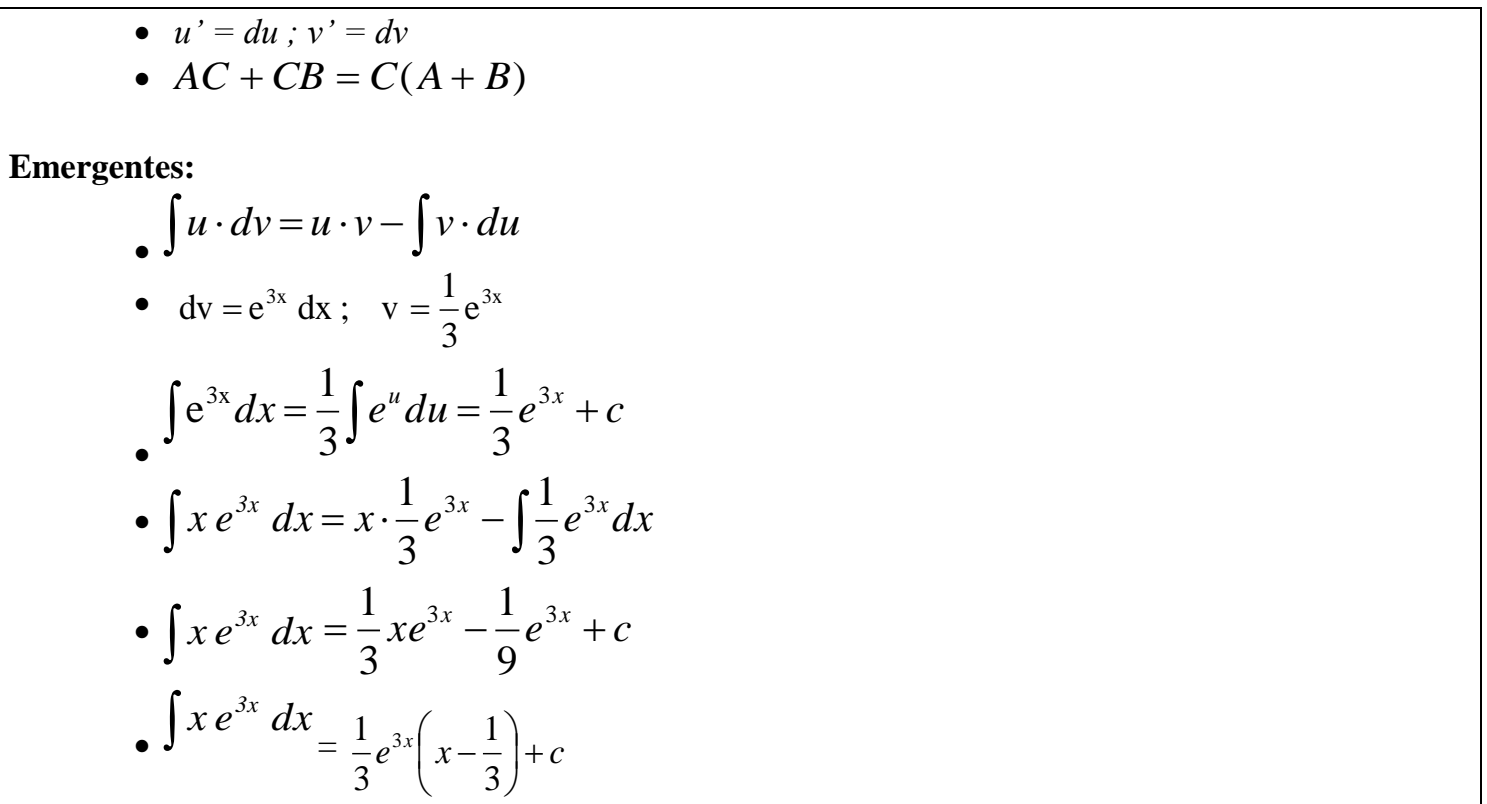 \\
\hline $\begin{array}{l}\text { Lenguaje: } \\
\text { Simbólico: } \\
e^{x}, u, v, d u, d v, u \text { ', } v \text { ', } f(u \cdot v), x, d x, \int \quad, C, \ldots . \\
\text { Verbal: "euler a la } 3 \mathrm{x} \text { " }\end{array}$ \\
\hline $\begin{array}{l}\text { Argumento 1. } \\
\text { Tesis 1: } \int u \cdot d v=u \cdot v-\int v \cdot d u \\
\text { Razón: Si tomamos la fórmula de la derivada de un producto, y si luego integramos a ambos lados de la } \\
\text { igualdad, simplificamos y obtenemos la igualdad. } \\
\text { Tesis 2: } \int x \mathrm{e}^{3 \mathrm{x}} \mathrm{dx}=\frac{1}{3} e^{3 x}\left(x-\frac{1}{3}\right)+c \\
\qquad \int x \mathrm{e}^{3 \mathrm{x}} \mathrm{dx} \text { es un caso donde se puede aplicar el método de integración por } \\
\text { Razón 1: Esta integral } \quad \text { partes donde hay un producto de funciones, donde una función no es la derivada de la otra. } \\
\text { Razón 2: Se ha aplicado el método de integración por partes a esta integral en particular y se ha } \\
\quad \text { obtenido el siguiente resultado } \frac{1}{3} e^{3 x}\left(x-\frac{1}{3}\right)+c\end{array}$ \\
\hline
\end{tabular}

\section{Cuadro 1- Configuración epistémica asociada a la CD2}

El análisis detallado de las prácticas hechas en la clase muestra que se ponen en juego muchos de los procesos matemáticos que considera el EOS (FONT; PLANAS; GODINO, 2010), además de otros. Dado que los procesos son densos en la actividad matemática, no pretendemos realizar un estudio exhaustivo de ellos; por ello, nos limitamos en esta sección a realizar una síntesis, tomando los más relevantes al mirar la CD2 de manera global. De modo general, el análisis indica que, esencialmente, se realiza un proceso de institucionalización del método de integración por partes y el proceso de algoritmización (mecanización) de dicho método. Por otra parte, hay que resaltar que se realizan procesos de tratamiento de expresiones simbólicas. Al discriminar los objetos (Cuadro 1) y procesos presentes en la práctica de la CD2 se pueden 
resaltar ciertos aspectos que con otro tipo de herramientas no se muestran tan claramente. Detallamos, a continuación, algunos de ellos:

- Los problemas que presenta el profesor: el tema, pertenecen estrictamente a un contexto intramatemático ${ }^{2} \mathrm{y}$, por tanto, no se fomentan procesos de modelización.

- Se presentan notaciones que no son institucionales y, por tanto, ambiguas para la investigación adelantada. Por ejemplo, $\mathcal{f}^{(u \cdot v)}$ o bien cuando se refiere a la función euler a la tres equis para referirse a la expresión $e^{3 x}$

- No hay argumentos; las explicaciones que se dan se limitan a ejemplificaciones del procedimiento a seguir.

- Las propiedades y procedimientos relevantes del tema (en este caso, los relacionados con el método de integración por partes o el Teorema Fundamental del Cálculo [TFC]) se presentan de manera ambigua. Por ejemplo, en el procedimiento para el cálculo de una integral por partes no se da el siguiente criterio para la selección de $u$ y $d v$ (para seleccionar $u$ en forma acertada hay que considerar la siguiente jerarquía: primero las inversas de las trigonométricas, logarítmicas, algebraicas, trigonométricas y exponenciales). Otro ejemplo es la enunciación del TFC (el proceso de antiderivar es el inverso a la derivada). Otro ejemplo, es que en lugar de enunciar la propiedad $A+C=B \Rightarrow A+C-C=B-C$ dice lo que está sumando pasa restando.

\section{Análisis de las trayectorias e interacciones didácticas}

Godino et al. (2006) describen, utilizando como criterio el tipo de interacción, cuatro tipos teóricos de configuraciones: magistral, a-didáctica, personal y dialógica. Las configuraciones reales que acontecen están más o menos próximas a ellas. Una CD se considera a-didáctica cuando el alumno y el maestro logran que el primero asuma el problema planteado como propio y entre en un proceso de búsqueda autónomo, sin ser guiado por lo que pudiera suponer que el maestro espera. La configuración teórica magistral se basa en la manera tradicional de enseñar matemáticas con exposición, seguida de ejercicios sobre los contenidos presentados. Una variante intermedia entre los tipos anteriores puede definirse cuando el profesor se encarga de la formulación y validación, mientras que los alumnos se responsabilizan de la exploración.

\footnotetext{
${ }^{2}$ Un contexto intra-matemático es aquel en el que la tarea específica a realizar se refiere solamente a objetos matemáticos tales como estructuras o símbolos. Mientras que un contexto extra-matemático incluye elementos externos que influyen en la interpretación y solución.
} 
La institucionalización tiene lugar mediante un diálogo entre el docente y los alumnos, quienes han tenido ocasión de asumir la tarea, familiarizarse con ella y, posiblemente, de esbozar alguna técnica de solución. En este caso, se habla de configuración teórica dialógica. Otro tipo teórico de CD surge cuando el estudiante resuelve la situación problema sin intervención directa del docente; aquí, los alumnos resuelven ejercicios propuestos por el profesor o que incluye el libro de texto. Se trata de un tipo de CD en la que predomina el estudio personal y que se denomina configuración didáctica personal. En la CD2 que hemos considerado para nuestro estudio, podemos notar que básicamente se enseña matemáticas con exposición, seguida de ejercicios sobre los contenidos vistos. Este modelo se repite en toda la secuencia de clases observada. Este modelo de enseñanza deja a los alumnos la responsabilidad de dar sentido a los objetos matemáticos que se introducen a través de los ejemplos y ejercicios que se van mostrando. Como expresan Godino et al. (2006, p. 31), se estaría tratando de una decisión topogenética: "primero yo, el profesor, te doy las reglas generales, después tú las aplicas". Aunque el registro de la sesión muestra un constante diálogo entre el profesor y los alumnos, que podría llevarnos a situar la clase en una configuración dialógica, un análisis más detallado revela que las interacciones se circunscriben a que los estudiantes asuman las tareas, se familiaricen con ellas y se esboce la técnica de resolución de integrales por el método de integración por partes. La institucionalización, formulación y validación quedan, exclusivamente, a cargo del profesor, sin intervención alguna de los alumnos, más allá de salir al tablero. Por dicho motivo, las configuraciones didácticas de toda la secuencia de clases se han considerado del tipo magistral-interactivo o bien de configuración didáctica personal.

Por otra parte, dada la gran diversidad de interacciones didácticas que pueden ocurrir en cualquier proceso de instrucción, nos hemos centrado en las que giran en torno a conflictos del tipo semiótico, de asequible individualización, ya que resulta fácilmente triangulable su identificación. Un conflicto semiótico en el EOS es cualquier disparidad o discordancia entre los significados atribuidos a una expresión por dos sujetos (personas o instituciones). Si la disparidad se produce entre significados institucionales se habla de conflictos semióticos de tipo epistémico, mientras que si se da entre prácticas que forman el significado personal de un mismo sujeto se llaman conflictos semióticos de tipo cognitivo (una noción muy relacionada con la de conflicto cognitivo propuesta por Piaget). Cuando la disparidad surge entre las prácticas - discursivas y operativas - de dos sujetos diferentes en interacción comunicativa (por ejemplo, alumno-alumno o alumno-profesor) se habla de conflictos semióticos interaccionales. Hay que notar que estos tres tipos de conflicto semiótico no son excluyentes, 
ya que, según la perspectiva desde donde se enfoque, un mismo conflicto puede ubicarse en un tipo u otro.

Es importante destacar que el análisis de cada uno de los conflictos puede comprenderse mejor si se tiene en cuenta la CD donde se enmarca, pues hay que relacionarlo con las prácticas matemáticas que se llevan a cabo en ella, los objetos y procesos involucrados en dichas prácticas y los patrones de interacción y las normas que intervienen en la CD.

A continuación, presentamos algunos de los conflictos semióticos más representativos que acontecen en la CD2.

- Conflicto semiótico (epistémico) 1: Tal como se observa en el Cuadro 1, el profesor, de manera implícita, institucionaliza un procedimiento para calcular integrales por el método de integración por partes que no contempla una paso esencial para seleccionar $u$ y $d v$ (no explica la jerarquía para dicha selección, según el tipo de función) ya que se limita a decir que $u$ será la primera y lo que queda será $d v$. Se trata de un conflicto muy relevante, ya que implica que en la CD4 los alumnos no puedan resolver la integral propuesta $\left(\int \mathrm{e}^{\mathrm{x}} \operatorname{sen} \mathrm{x} \mathrm{dx}\right)$, lo cual también sucede con otras integrales en clases 3-12 descritas en la unidades de análisis [123-820] reitero, que por razones de espacio, se omiten aquí.

- Conflicto semiótico (cognitivo) 1: en la sesión 1 CD2 [35] el profesor institucionaliza que al existir una multiplicación entre funciones no similares, el método a aplicar es por partes. Estamos en presencia de un conflicto cognitivo que el profesor genera en el alumno. Este conflicto se hace evidente en la sesión 2 CD5 [231] ante la imposibilidad de resolver la integral $\int \frac{\operatorname{sen} 2 x}{e^{x}} d x$ A13 dice que no se puede aplicar la integración por partes porque no hay un producto de funciones, el profesor resuelve este conflicto ayudándole a avanzar en su aprendizaje indicándole que si se hace el siguiente tratamiento $\int e^{-x} \operatorname{sen} 2 x d x$, sí que se tiene un producto de funciones.

- Conflicto semiótico (cognitivo) 2: del conflicto epistémico mencionado, se observa la creación de un conflicto de tipo cognitivo en los estudiantes. En la sesión 1 CD2 [130-132] el profesor genera este conflicto cognitivo en el alumno cuando, ante el ejercicio propuesto $\int x \operatorname{sen} x d x$, aprueba la respuesta de un estudiante ante la pregunta ¿quién será $u$ ? A9 responde: $x$, el Prof. pregunta ¿Por qué?, los estudiantes responden porque esta de primeras. Los alumnos se quedan pensando que $u$ siempre será la función que aparece en el registro escrito de primeras. Se observa la imposibilidad de 
elegir a $u$ en forma correcta. Este conflicto es relevante, no es superado. Otro ejemplo se da en la sesión 1. CD4, que por cuestión de espacio no se muestra en este escrito, ante el ejercicio propuesto $\int e^{x} \operatorname{sen} x d x$, donde los estudiantes eligen a la función $e^{x}$ como $u$ y $\operatorname{sen} x d x$ como $d v$, llegando a inconsistencias en el desarrollo del ejercicio propuesto. haciendo procesos mecánicos, repetitivos, sin validar la respuesta obtenida. La misma situación se presenta, más adelante, en la sesión 1 CD5, que también por cuestión de espacio no se muestra en este escrito, con el ejercicio $\int \frac{\operatorname{sen} 2 x}{e^{x}} d x$ manifestando la imposibilidad de aplicar este método por dos razones: la primera, no existe un producto entre sus términos; luego del tratamiento que el profesor presenta para expresar la integral en la forma $\int e^{-x} \operatorname{sen} 2 x d x$ los estudiantes vuelven a elegir como $u$ a la función $e^{-x}$ llegando a resultados similares al expuesto anteriormente. En general, este conflicto se hace evidente en todas las 12 sesiones de clase.

- Conflicto semiótico (interaccional) 1: En [81-90] el alumno A13 dice que la solución de $\int e^{3 x} d x$ es $e^{3 x}$ y el alumno A6 que es $\int \mathrm{e}^{3 \mathrm{x}} d x=\frac{1}{3} \mathrm{e}^{3 \mathrm{x}}$ otra diferente. Este conflicto se resuelve bien por la intervención de A12 que le explica a A13 porque está equivocado

- Conflicto semiótico (interaccional) 2: En $[152-158]^{3}$ el alumno A12 duda de la solución que se está dando al ejercicio $\int e^{x} \operatorname{sen} x d x$ al notar que la integral que resultó no era más sencilla que la propuesta en el ejercicio. Ante la observación de A12, el profesor borra del tablero lo que han escrito y hace el cambio en la selección de $u$ y $d v$. repite el proceso interactivo para llegar a $\int e^{x} \operatorname{sen} x d x=-e^{x} \cos x-\int-e^{x} \cos x d x$ en [157] A12 reitera la duda en la solución planteada, que conlleva a la no comprensión de este tipo de solución. Este conflicto no se resuelve por la intervención del profesor, queda latente ya que la clase se termina y tampoco se aborda en las sesiones siguientes.

\section{Identificación del sistema de normas y metanormas}

El sistema de normas que regulan la interacción del aula, ha sido objeto de investigación en Didáctica de las Matemáticas, principalmente por Blumer (1969),

\footnotetext{
${ }^{3}$ Es importante relacionarlo en este apartado, a pesar que por cuestión de espacio en este escrito no se mostrará.
} 
introduciendo nociones como patrones de interacción, normas sociales y sociomatemáticas (COBB; BAUERSFELD, 1995; YACKEL; COBB, 1996); y mediante la noción de contrato didáctico (BROUSSEAU, 1988). En ambos casos, se trata de tener en cuenta las normas, hábitos y convenciones generalmente implícitas que regulan el funcionamiento de la clase de matemáticas, concebida como microsociedad, que condicionan, en mayor o menor medida, los conocimientos que construyen los estudiantes. El foco de atención, en estas aproximaciones, ha sido principalmente las interacciones entre profesor y estudiantes cuando abordan el estudio de temas matemáticos específicos.

Por una parte, se tiene que los elementos matemáticos asociados con la solución del problema corresponden a un curso del nivel de Licenciatura en Matemáticas y se encuentran descritos en el currículo y los libros de texto de las materias que cursan los estudiantes de la Facultad de Ciencia y Tecnología. Por otra parte, el diseño curricular no favorece la enseñanza con base en problemas. Los cursos están concebidos en forma expositivoilustrativa, con tareas y actividades específicas propias de esa modalidad. Algunas de las normas que se infieren de las regularidades que se observan en CD2 y en las otras configuraciones son las siguientes: 1) Los ejercicios de matemáticas se hacen de determinada manera (metanorma metaepistémica). Por ejemplo, en [35, 36, 65, 133,]. 2) Para aprender matemáticas hay que hacer muchos ejercicios (norma metacognitiva). Por ejemplo, en [120, 434...]. 3) Hay que dedicar mucho tiempo a hacer ejercicios (norma mediacional). Por ejemplo, en [120, 433...]. Otras normas que regulan las interacciones y que, implícitamente, aparecen en las clases, son, entre otras, las siguientes: el profesor interviene para resolver algunas dificultades de los alumnos; otras son resueltas por otros compañeros; el profesor tiene un papel determinante en el inicio, distribución y finalización de las intervenciones; algunos alumnos participan cuando no entienden algo.

\section{Valoración de la idoneidad didáctica del proceso de instrucción}

La aplicación de los criterios de idoneidad a un proceso de enseñanza y aprendizaje permiten extraer conclusiones sobre qué aspectos mejorar en futuras implementaciones.

\subsection{Idoneidad epistémica}

Del análisis de los registros de la clase se evidencia que el profesor ha trabajado únicamente con ejercicios en contexto intramatemático, ninguno de ellos puede considerarse 
como un problema, y no hay situaciones que sean generadoras de problemas, excepto cuando el profesor propone buscar una regla que permita resolver las integrales que no se pueden resolver por sustitución (falta de procesos de modelización y problematización). Las actividades que propone no motivan los momentos en que los alumnos tengan que hacer conjeturas ni justificaciones. Incluso cuando pudo ser posible para buscar la regla de integración por partes, es el profesor quien termina encontrando la regla y justificándola. Por otra parte, el profesor no justifica suficientemente cuándo hay que aplicar la regla de integración por partes y cuándo la sustitución. [ej. Unidad 23. "Este es un ejercicio que tiene forma racional, pues tiene Polinomios arriba y abajo. El de abajo es un radical polinómico. Entonces María, recuerde que se debe mirar bien para descubrir la forma de calcular la integral. Tenga en cuenta revisar cual es la derivada del otro. ¿Cuál sería u?”. Otras situaciones similares se pueden evidenciar en las unidades de análisis 24 al 35, 36, 45,51-52, 58, 67, 113, 128-132, 317 y 318, 323, 372 y 373]. En esta débil justificación, hay que resaltar la presencia de muchas ambigüedades como puede ser el uso de arriba y abajo en lugar de decir numerador y denominador.

Los procesos de simbolización que realiza el profesor, en algún caso, son ambiguos por no decir incorrectos (por ejemplo, utiliza símbolos que no son institucionales como tachar la integral y la prima $\mathfrak{J}^{(u \cdot v)}$ ). Si bien se observan tratamientos no se institucionaliza la importancia de este tratamiento. Y, por otra parte, no se observan conversiones ${ }^{4}$. No se fomentan las conexiones entre la integración por partes y los problemas extra-matemáticos donde se puede aplicar, pero tampoco se tiene mucho cuidado con las conexiones intramatemáticas. Ejemplo: la justificación que da el profesor de la igualdad en la que se basa la integración por partes no está bien conectada con la idea de antiderivada, ya que puede sugerir al alumno que la antiderivada no es una familia de funciones, sino una única función. El proceso fundamental que se da es el la algoritmización, pero de manera imprecisa, ya que la práctica que realizan los alumnos durante las nueve últimas sesiones resolviendo muchas intégreles por partes no les lleva a tener claros los pasos del método de integración en particular el orden jerárquico para seleccionar la $u$.

\footnotetext{
${ }^{4}$ Según Duval (2006) las transformaciones semióticas y la coordinación entre los registros de representación son imprescindibles en la actividad matemática. Las transformaciones pueden ser clasificadas en dos tipos: tratamientos y conversiones. La distinción entre esos dos tipos de registro posibilita analizar el funcionamiento del sistema cognitivo de comprensión del sujeto. Duval (2006) destaca que es a través de la coordinación entre los registros lo que permite la adquisición de conocimientos. Nos afirma que, "la comprensión de un contenido conceptual reposa sobre la coordinación de al menos dos registros de representación, y esa coordinación se manifiesta por la rapidez y espontaneidad de las actividades de conversión” (DUVAL, 2006, p. 113).
} 
De las diferentes configuraciones epistémicas para modelizar la complejidad de la integral, descritas en Ordoñez (2011) solo se usan parcialmente la configuración que llaman inversa de la derivada y la algebraica. Por otra parte, con relación a estas dos configuraciones, los elementos esenciales no están bien explicados ni coherentemente organizados (el procedimiento de integración por partes es ambiguo, olvida un paso fundamental que es la jerarquía para la selección de $u$, el TFC se expresa de forma ambigua etc). No hay una muestra representativa de los tipos de problemas en donde es indicado aplicar el método de la integración por partes; incluso el tipo de problemas donde se debe aplicar dos veces el mismo método, no les hace observar que hay una familia de problemas que se resuelven por integración repetida del algoritmo. El Cuadro 2 evidencia con $x$ esta falta de representatividad de los problemas propuestos ya que la clase se reduce a ejercicios del tipo marcado con $x$.

\begin{tabular}{|c|r|c|c|c|c|}
\hline Tipo de ejercicios & I.T. & Log. & Alg. & Trig. & Exp. \\
\hline Inversas de trigonométricas (IT) & & $x$ & & $x$ & \\
\hline Logarítmicas (Log) & $x$ & & $x$ & $x$ & \\
\hline Algebraicas (Alg) & $x$ & $\mathbf{x}$ & & $\mathbf{x}$ & $\mathbf{x}$ \\
\hline Trigonométricas (Trig) & & $x$ & $\mathbf{x}$ & & $\mathbf{x}$ \\
\hline Exponenciales (Exp) & $x$ & $x$ & $\mathbf{x}$ & $\mathbf{x}$ & \\
\hline
\end{tabular}

Cuadro 2 -Situaciones problema propuestos por el profesor Fuente: Elaboración propia

No se presentan ejercicios que involucren los productos de funciones: inversas de trigonométricos por logarítmicas. No considera ejercicios que involucren más de una configuración epistémica. La falta de procesos relevantes, en particular de modelación y de justificación; la falta de representatividad de significados parciales (solo la configuración inversa de la derivada y algebraica); la falta de variedad de problemas donde se aplica la integración por partes; la existencia de ambigüedades en la explicación de los elementos esenciales y en su organización, etc. Llevan a considerar que estas clases tienen una baja idoneidad epistémica. De acuerdo con Font y Godino (2006) consideramos que las matemáticas implementadas en estas clases se pueden considerar como unas matemáticas mecanicistas, que son una degeneración de las formalistas. La configuración epistémica de esta clase termina siendo una distorsión mecanicista de una formalista, ya que aparecen algunos aspectos poco deseables de este último tipo de configuraciones (descontextualización y falta de procesos de abstracción) y desaparecen los aspectos más deseables (precisión, coherencia lógica). Las matemáticas implementadas presentan las siguientes características:

- Se propone una amplia lista de ejercicios del texto guía para realizarlos en forma mecánica, de manera descontextualizada. 
- Se realizan presentaciones ambiguas del contenido matemático y no se organizan de manera coherente.

- No se contemplan las conversiones entre diferentes formas de representación.

- El profesor sigue un modelo de clase magistral.

- La argumentación es casi inexistente. Se abandonan las demostraciones y no son sustituidas por justificaciones de tipo inductivo, grafico, visual, abductivo etc.

- Se manipulan símbolos, sin dotarles de significación.

- No se emplean situaciones de referencia que le den sentido a las matemáticas enseñadas, ya que se presentan unas matemáticas centradas sobre ellas mismas y muy alejadas de las otras ciencias.

\subsection{Idoneidad cognitiva}

La baja idoneidad epistémica que presenta la clase ayuda a que los contenidos enseñados estén a una distancia razonable de lo que saben los alumnos. Por otra parte, la mayoría de los estudiantes aprendió lo que el profesor pretendía enseñar y realmente enseñó. El registro de clase pone en evidencia que los alumnos tienen un manejo adecuado de la operatoria aritmética, un mediano manejo de la operatoria algebraica, un requisito básico para iniciar el estudio del cálculo integral si se piensa como aritmética generalizada (la mayor parte de las expresiones y manipulaciones algebraicas pueden ser explicadas a partir de las expresiones y manipulaciones aritméticas). Así, se observa que resuelven integrales correctamente, pero sólo adquieren un conocimiento instrumental que les permite hallar una solución sin saber por qué se resuelven de esta manera y no de otra. Sostenemos que la enseñanza de un contenido matemático no sólo debe pensarse que términos de que esté al alcance de las capacidades cognitivas con que cuentan los alumnos, sino también debe promover el desarrollo de nuevas competencias, las cuales suponen un cierto reto cognitivo manejable. Esta situación precisamente no se ve reflejada en la clase.

\subsection{Idoneidad mediacional}

El número de alumnos (20 en total) y su distribución en el aula permite llevar a cabo el proceso instruccional adecuadamente. No obstante, durante la clase no se hace uso de materiales manipulativos e informáticos que permitan introducir buenas situaciones, 
lenguajes, procedimientos o argumentaciones adaptadas al significado institucional de referencia. Sólo se plantea la temática con el empleo de un lenguaje simbólico, sin apoyo en modelos concretos o visualizaciones; por lo tanto, las definiciones y propiedades emergen descontextualizadas. Prácticamente todo el tiempo invertido en la sesión de la clase se circunscribe a la enseñanza de una técnica particular de resolución de integrales por el método designado, y no se evidencian configuraciones didácticas donde el profesor tenga en cuenta las potenciales dificultades de comprensión de los alumnos. Por otra parte, consideramos que un primer acercamiento a la enseñanza del cálculo integral nos lleva a movernos en un marco donde las representaciones son relativas a un sistema particular de signos y pueden ser convertidas en representaciones equivalentes en otro sistema semiótico, pero toman significaciones diferentes para el sujeto que las utiliza.

El análisis de los objetos, procesos y trayectorias epistémicas de la clase nos indica que el profesor pareciera hacer caso omiso a la necesidad de construir un nuevo lenguaje para poder integrar el aprendizaje del cálculo integral a los dominios cognitivos de los alumnos. En este sentido, el profesor pareciera adoptar la posición antagónica que describe Filloy (1999), bajo el modelo sintáctico-viético, cuando propone a los alumnos partir del nivel sintáctico y enseñar sus reglas para aplicarlas más tarde en la resolución de ecuaciones y problemas. Todo lo anteriormente dicho nos lleva a decir que el proceso de instrucción es de baja idoneidad mediacional.

\subsection{Idoneidad afectiva}

En el registro de la clase se evidencia que el profesor busca favorecer la argumentación en situaciones de igualdad, ya que valora el argumento en sí mismo y no por quién lo dice. De todos modos, no se proponen situaciones que permitan calificar la utilidad de las matemáticas en la vida cotidiana y profesional. Además, no hay procesos de personalización, pues aunque los estudiantes se involucran inicialmente en la actividad, comienzan a perder interés, en varios momentos de la clase, y dejan de sentir interesante lo que se les propone, ya que se desorganizan al sentir que se pierden en el proceso a seguir para solucionar el ejercicio, lo que el profesor hace es pasar al tablero a otros estudiantes a solucionar el ejercicio, hecho que desmotiva a otros estudiantes que no se involucran a la clase, otros preguntan cuánto falta para terminar con la hora etc. La presencia de normas y metanormas del tipo: 1) para comprender determinados temas en matemáticas se ha de esperar a los cursos superiores y 2) se deben hacer mediante ejercicios largos (por eso son bellas y 
difíciles), y 3) para aprender matemáticas hay que hacer muchos ejercicios; dan un marco apropiado para el surgimiento de conflictos semióticos que no se resuelven, manifestados en los alumnos por sentimientos de rechazo, fobia, ansiedad y temor hacia el estudio de esta disciplina. Así, algunos de ellos llegan a considerar a las cuestiones de las matemáticas como demasiado complejas y fuera de su alcance. [Unidades de análisis 155, y las mencionadas en la idoneidad cognitiva] En consecuencia, el proceso de instrucción muestra una idoneidad afectiva baja.

\subsection{Idoneidad interaccional}

La clase presenta objetivos instruccionales claros y observables, pero la enseñanza se realiza por imitación y asociación, y por refuerzos/castigos. En consecuencia, el aprendizaje se produce por observación de lo que hace un experto, mientras que el alumno es receptor del proceso y sigue instrucciones. Los momentos de institucionalización del conocimiento matemático no son producto de fases de discusión sobre los aspectos críticos del proceso de aprendizaje. Así, la topogénesis del conocimiento y la construcción misma del saber se encuentra del lado del profesor, quien privilegia unas experiencias educativas en detrimento de otras. Los patrones de interacción, la manera de resolver los conflictos y la presencia de metanormas del tipo los ejercicios de matemáticas se hacen de determinada manera puede encaminar a que los alumnos piensen que existe sólo un algoritmo apropiado que garantiza la respuesta. Por otra parte, la creación de conflictos por parte del profesor, y la no resolución satisfactoria de la mayoría de los que provienen de los alumnos, genera un tipo de comprensión no significativa en los alumnos, documentada en muchas investigaciones, como las relacionadas por Artigue, Batanero y Kent, 2010) y Cordero (2005), entre otras. Por otra parte, el análisis de las trayectorias e interacciones didácticas nos orienta a situar esta clase en el tipo magistral interactivo. Es magistral porque se otorga autoridad al profesor, considerado experto, que se sitúa en un estatus superior al del destinatario, permitiendo que gestione su discurso y que imponga unas normas aceptadas por los estudiantes.

En este caso, es innegable la autoridad del profesor porque goza de prestigio del saber que le concede su rol profesional, y tiene poder institucional que se traduce en aprobar y en reprobar. Es interactiva porque se acepta la incorporación de otras voces en el discurso (aunque participa un grupo reducido de jóvenes estudiantes), pero prevalece una interacción monológica entre docente y discentes, ya que están presentes preguntas cerradas y específicas del profesor que requieren de la respuesta convergente y fáctica de los alumnos. A su vez, el 
profesor acepta como válidas las respuestas, en la medida que coincidan con el discurso previamente diseñado. La situación descripta nos lleva a considerar que el proceso de instrucción es de baja idoneidad interaccional.

\subsection{Idoneidad ecológica}

Si bien la Facultad de Ciencia y Tecnología a la que está adscrita la Licenciatura en Matemáticas establece lineamientos generales, es claro que el proceso de instrucción analizado no sigue las directrices curriculares. Una presentación del cálculo integral basada en la sola ejecución de las reglas de transformación de términos a través de ejemplos desarticulados impide que se incorporen relaciones con otros contenidos intra e interdisciplinares. Además, suele fomentar en los alumnos una concepción de las matemáticas como algo perfecto y acabado que consiste en memorizar y aplicar un conjunto de reglas. En síntesis, el proceso de instrucción es de baja idoneidad ecológica. En el siguiente diagrama (Figura 1) suponemos el hexágono regular exterior como la idealización de la idoneidad didáctica de un proceso de instrucción de buena calidad (se consiguen de manera conjunta todas las idoneidades), mientras que el hexágono interno refleja la idoneidad de la clase observada.

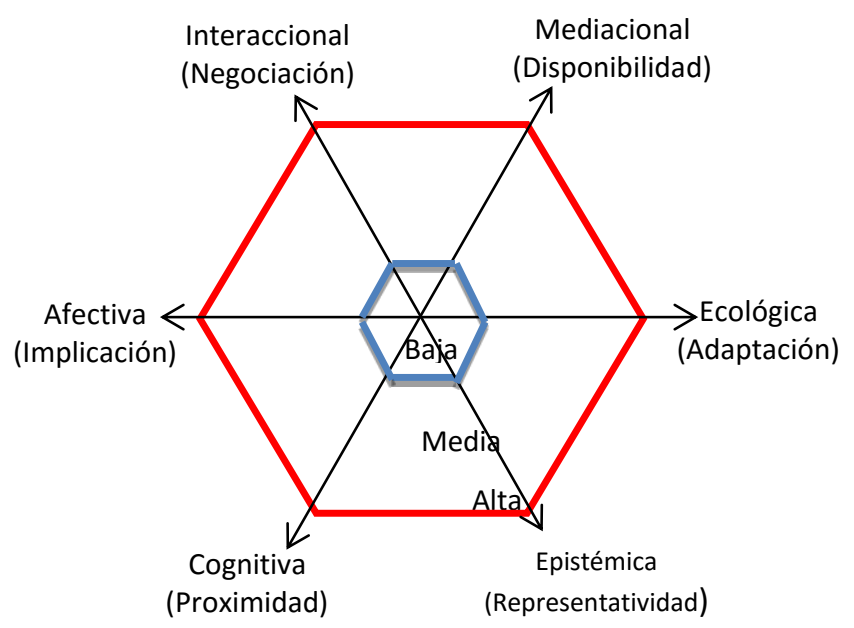

Figura 1 - Idoneidad didáctica del proceso de instrucción Fuente: Robles, Castillo, Font, 2012

\section{Dificultades observadas y su explicación a partir del análisis didáctico realizado en los apartados anteriores}


Se observan tres grandes dificultades a lo largo de las 12 sesiones de clase utilizadas para implementar el proceso de instrucción del método de integración por partes (algunas de ellas presentes desde la sesión 1 en la $\mathrm{CD} 2$, centro de este artículo y que se vuelven reiterativas a lo largo de las doce sesiones observadas), que no se superan. En su orden son:

1. Dificultad para determinar el orden jerárquico para elegir cuál función se nombrará como $u$ y cuál como $d v$. Conduciendo a los estudiantes a considerar que siempre debe tomarse como $u$ la función que está escrita de primero en la integral, llevándolos a cometer errores que no se superan a lo largo de las sesiones de clase observadas.

2. Dificultad para hacer transformaciones del tipo: tratamientos, conversiones, trasferencias y aplicar la regla de integración por partes. Aspectos que si se consideraran permitirían superar la primera dificultad mencionada en el párrafo anterior.

3. Dificultad para solucionar problemas pertenecientes a otros contextos científicos como la física y la economía, por nombrar algunos. Dado que la clase se limita a desarrollar, únicamente, algunos ejercicios propuestos en el libro de texto de la bibliografía; muchas veces excluyendo los problemas propuestos en contexto.

\section{Consideraciones finales}

En este trabajo hemos aplicado un modelo que permite realizar un análisis didáctico sistemático para la descripción, explicación y valoración de episodios de clases de matemáticas. La descripción de la sesión de clase es el resultado de una metodología de observación, que ha consistido en aplicar los constructos del marco teórico adoptado. Dicho marco nos ha servido de guía sobre lo que había que observar, cómo se debía observar y nos ha proporcionado las herramientas para realizar la observación.

Hemos mostrado, además, cómo un análisis minucioso, apoyado en las herramientas didácticas que provee el EOS, precisa e ilustra con detalles la estructura y funcionamiento de una clase de matemáticas que se puede considerar como una degeneración mecanicista de una clase formal ${ }^{5}$. Los diferentes niveles de análisis permiten diferenciar todo lo que está

\footnotetext{
${ }^{5}$ Dado que en el desarrollo de la misma se utilizan características propias del paradigma formal-mecanicista. Entre ellas: supone que todo sistema social está organizado en niveles, estructurados en formas jerárquicas a través de relaciones de poder unidireccionales que para este caso recaen en el profesor. Existe un abordaje prescriptivo de la realidad organizacional, mediante la aplicación de reglas y normas que regulan su funcionamiento (aplicación reiterativa de algoritmos en forma descontextualizada para el estudiante). Se presenta, constantemente, un estímulo-respuesta para explicar conductas individuales y organizacionales (presentación de algoritmos como algo dado, que no es necesario construir, deducir de dónde o cómo se llega a
} 
involucrado en el conglomerado que conforma una clase de matemáticas (situación problema, lenguaje, conceptos, proposiciones, procedimientos y argumentos, interacciones, conflictos, normas y metanormas), así como establecer relaciones entre dichas partes. En términos metafóricos, podemos decir que se obtiene una radiografía de la clase, donde se observan conflictos semióticos que están relacionados con dificultades de los alumnos evidenciadas en sus exámenes, que además han sido documentados en otras investigaciones.

La enseñanza de las integrales es un tópico ampliamente estudiado en la Matemática Educativa. Un grupo relevante de estos trabajos, que normalmente ocupan marcos teóricos de tipo psicológico, han investigado las dificultades que presentan los alumnos con la aplicación de cuestionarios y entrevistas, pero no analizan los procesos de instrucción que siguen los estudiantes. En cambio, el análisis que hemos llevado a cabo aquí, además de permitir hacer una descripción con detalle lo que ha sucedido, ofrece explicaciones sobre porqué se presentan determinadas dificultades en los alumnos. Por una parte, podemos afirmar, como muestra la investigación sobre la didáctica de las integrales, que los alumnos tienen problemas para entender la resolución de integrales por el método de integración por partes, y que se observan, entre otras evidencias, a través de los conflictos semióticos descritos en el análisis de la transcripción. Por otra parte, se pueden hacer predicciones sobre lo que probablemente sucederá en un proceso de enseñanza y aprendizaje de las matemáticas cuya radiografía sea parecida: se producirán conflictos semióticos parecidos que no serán resueltos y generarán dificultades. Dicho en forma breve, este tipo de clase que es una degeneración mecanicista de una clase formalista es una de las causas de la generación de determinadas dificultades; sin embargo, cabe resaltar que el mecanismo causal es su estructura y funcionamiento.

Aunque no se ha hecho en este trabajo, por cuestiones de espacio, se puede profundizar en la relación entre este tipo de clases y las dificultades de comprensión. Si se realiza un análisis Ontosemiótico a priori que ponga de manifiesto las diferentes configuraciones epistémicas que forman el significado de referencia del objeto integral, y la trama de funciones semióticas que se han de activar para relacionar entre sí los elementos de las configuraciones y las configuraciones entre ellas - como el realizado en Contreras, Ordóñez y Wilhelmi (2010); Crisóstomo (2012); Ordóñez (2011); Robles, Telechea y Font (2014), se puede observar, en la transcripción, que las configuraciones didácticas implementadas no han tenido en cuenta dicha complejidad. El análisis, a priori, nos permite decir que la estructura y funcionamiento de este

esa construcción universalmente ya reconocida y aceptada). Tomar solo lo observable como objeto de estudio de la organización. Entre otras. 
tipo de clases no repara en la complejidad ontosemiótica asociada a las integrales, lo cual es una de las causas de que se produzcan determinadas dificultades.

La aplicación de los criterios de idoneidad a un proceso de enseñanza y aprendizaje concreto la hemos entendido como la metodología que permite la guía, valoración y posible mejora de un proceso de enseñanza y aprendizaje. En nuestro trabajo, estos criterios llegaron a ser reglas de corrección útiles en dos momentos: a priori, pues son principios que orientan cómo se deben hacer las cosas, y a posteriori, porque sirven para valorar el proceso de instrucción efectivamente implementado. Así, la aplicación de los criterios de idoneidad nos permitieron extraer conclusiones sobre qué aspectos mejorar en el futuro. Por ejemplo, la baja idoneidad epistémica de la clase se debería mejorar.

Finalmente, destacamos que este análisis didáctico minucioso, al igual que una radiografía, penetra en la estructura interna de la clase, resaltando aspectos y matices que, si bien pueden parecer obvios después de haber sido encontrados, se hallan ocultos ante una mirada general y prematuramente valorativa de esta práctica matemática. Nuestra conclusión es que el análisis hecho resulta útil en dos aspectos. Por una parte, como se afirma en Font, Planas y Godino (2010), el modelo de análisis didáctico aplicado en este trabajo puede ser útil para el colectivo de profesores interesados en reflexionar sobre su propia práctica. Por otra parte, la radiografía de la clase mecanicista puede orientar a los profesores en formación y a los que imparten este tipo de clase a valorar, reflexionar y sugerir acciones de mejora.

\section{Referencias}

ARTIGUE, M.; BATANERO, C.; KENT, P. Mathematics thinking and learning at post secondary level. In.. LESTER, F.(Ed.), Second Handbook of research on Mathematics Teaching and learning. Charlotte, NC: IAP - Information Age Publishing. 2010. p. 1011-1045.

BLUMER, H. Symbolic interactionism: Perspective and method. Nueva Jersey: Prentice Hall, 1969.

BROUSSEAU, G. Didactique fondamentale, in Didactique des mathématiques et formation des maîtres à l'école élémentaire. Actes de l'université d'été, Publication de l'I. R. E. M. de Bordeaux, 1988.

CONTRERAS, A.; ORDÓÑEZ, L.; WILHELMI, M. Influencia de las pruebas de acceso a la universidad en la enseñanza de la integral definida en el bachillerato. Enseñanza de las ciencias. Universitat Autònoma de Barcelona: Institut de Ciències de l'Educació, ICE. España. v. 28 (3), p. $367-$ $384,2010$.

CORDERO, F. El rol de algunas categorías del conocimiento matemático en educación superior. Una socio epistemología de la integral. Relime. México, v. 8 (3), p. 265-286, 2005.

COBB, P.; BAUERSFELD, H. The emergence of mathematical meaning: Interaction in classroom cultures. Hillsdale: Lawrence Erlbaum Associates, 1995. 
CRISÓSTOMO, E. Idoneidad de procesos de estudio del cálculo integral en la formación de profesores de matemáticas: una aproximación desde la investigación en didáctica del cálculo y el conocimiento profesional. 2012. p. 137-159, Universidad de Granada, Granada,2012.

DAVIS, B.; RENERT, M. Profound understanding of emergent mathematics: broadening the construct of teacher' disciplinary knowledge. Educational Studies in Mathematics Education. New York, v. 82 (2), p. 245-265, 2013.

DUVAL, R. A. Cognitive Analysis of Problems of Comprehension in a Learning of Mathematics. Educational Studies in Mathematics. ISSN: 0013-1954 (Print) 1573-0816 (Online), p. 103-13,.2006.

FERNÁNDEZ, C.; YOSHIDA, M. Lesson study: a Japanese approach to improving mathematics teaching and learning. Mahwah: Erlbaum. 2004.

FILLOY, E. Aspectos Teóricos del álgebra Educativa. México: Grupo Editorial Iberoamérica, 1999.

FONT, V.; BOLITE, J.; ACEVEDO, J. Metaphors in mathematics classrooms: analyzing the dynamic process of teaching and learning of graph functions. Educational Studies in Mathematics. Published online, Springer Science+Business Media B.V., v. 75 (2), p. 131-152, 2010.

FONT, V.; GODINO, J. La noción de configuración epistémica como herramienta de análisis de textos matemáticos: su uso en la formación de profesores. Educação Matemática Pesquisa, Brasil, ISSN: 1983-3156, v. 8 (1), p. 67-98, 2006.

FONT, V.; PLANAS, N.; GODINO, J. Modelo para el análisis didáctico en educación matemática. Infancia y Aprendizaje. Salamanca, España, v. 33 (1), p. 89-105, 2010.

GODINO, J. Un enfoque ontológico y semiótico de la cognición matemática. Reserches en Didactiques des Mathematiques. Francia, v. 22 (2/3). p. 237-284, 2002.

GODINO, J.; BATANERO, C.; FONT, V. Un enfoque ontosemiótico del conocimiento y la instrucción matemática. Departamento de Didáctica de la Matemática. Universidad de Granada, 2006. Disponible en: <http://www.ugr.es/local/jgodino/indice_eos.htm>. Visitado en: 10, 04, 2012. GODINO, J.; BENCOMO, D.; FONT, V.; WILHELMI, M. Análisis y valoración de la idoneidad de procesos de estudio de las matemáticas. Paragima. Huesca (España), v. 27 (2), p. 221-252, 2006.

GODINO, J.; CONTRERAS, A.; FONT, V. Análisis de procesos de instrucción basado en el enfoque ontológico-semiótico de la cognición matemática. Recherches en Didactiques des Mathematiques. Francia, v. 26 (1), p. 39-88, 2006.

GODINO, J; FONT, V.; WILHELMI, M.; LURDUY, O. Why is the learning of elementary arithmetic concepts difficult? Semiotic tools for undertanding the nature of mathematical objects. Educational Studies in Mathematics. Disponible en: <http://www.ugr.es/ jgodino/eos/JDGodino-semiotictools_ESM_2011.pdf>. Visitado en: 2, 06, 2013.

MASON, J. Researching your own practice. The discipline of noticing. London: Routledge-Falmer, 2002.

ORDOÑEZ, L.; WILHELMI, M. Influencia de las pruebas de acceso a la Universidad en la enseñanza de la integral definida en el Bachillerato. Enseñanza de las Ciencias, Barcelona, v. 28 (3), p. 367-384,2010.

ORDOÑEZ, L. Restricciones institucionales en las matemáticas de $2^{\circ}$ de bachillerato en cuanto al significado del objeto integral definida. 2011. p. 86-128. tesis doctoral- Matemáticas de $2^{\circ}$ de Bachillerato- Universidad de Jaén, España, 2011. 
POCHULU, M.; FONT, V. Análisis del funcionamiento de una clase de matemáticas no significativa. Revista Latinoamericana de Investigación en Matemática Educativa - RELIME. México, v. 14 (3), p. 361-394, 2011.

POCHULU, M.; FONT, V.; RODRIGUEZ, M. Desarrollo de la Competencia en Análisis Didáctico de Formadores de futuros Profesores de Matemática a traves del diseño de tareas. Revista

Latinoamericana de Investigación en Matemática Educativa - RELIME. México, p. 71-98, 2015.

ROBLES, G.; DEL CASTILLO, A.; FONT, V. Análisis y valoración de un proceso de instrucción sobre la derivada. Educación Matemática. México, v. 24 (1), p. 35-71, 2012.

ROBLES, M.; TELECHEA, E.; FONT, V. Una propuesta de acercamiento alternativo al teorema fundamental del cálculo. Educación Matemática. México, v. 26 (2), p. 69-109, 2014.

ROWLAND, T.; HUCKSTEP, P.; THWAITES, A. Elementary teachers' mathematics subject knowledge: the knowledge quartet and the case of Naomi. Journal of Mathematics Teacher Education. v. 8 (3), p. 255-281, 2005. Disponible en: < http://link.springer.com/article/10.1007\%2Fs10857-005-0853-5\#/page-1>. Visitado en: 12, 09, 2013.

YACKEL, E.; COBB, P. Sociomathematical norms, argumentation, and autonomy in mathematics. Journal for research in Mathematics Eduaction, EE.UU, v. 27 (4), p .458-477, 1996.

Submetido em Abril de 2015. Aprovado em Novembro de 2015. 


\section{Anexo 1 - Transcripción en unidades de análisis de la configuración didáctica 2}

\begin{tabular}{|c|c|c|c|}
\hline $\begin{array}{l}\text { \# de } \\
\text { und }\end{array}$ & & Descripción & Comentario del observador investigador \\
\hline 33 & $\mathrm{P}$ & $\begin{array}{l}\text { Vamos a la lección } 7.1 \text { del libro. ¡Vamos a calcular } \\
\text { la integral de equis, Euler a la tres equis de equis! }\end{array}$ & $\begin{array}{l}\text { [El profesor se acerca a su escritorio, allí tiene el } \\
\text { libro abierto en la sección que mencionó y } \\
\text { escribe en el tablero: ] } \\
\int x e^{3 x} d x\end{array}$ \\
\hline 35 & $\mathrm{P}$ & $\begin{array}{l}\text { ¡Bueno, si ven, este ejercicio tiene una multiplicación } \\
\text { entre sus términos, entonces el ejercicio se debe hacer } \\
\text { por partes! Así se llama la técnica que aprenderemos } \\
\text { hoy. }\end{array}$ & \\
\hline 36 & $\mathrm{P}$. & $\begin{array}{l}\text { Después que revisemos que no es posible factorizar los } \\
\text { términos para simplificar la expresión, verificamos si } \\
\text { la solución es por sustitución o en caso contrario será } \\
\text { por partes. Ustedes ya saben determinar si es por el } \\
\text { método de sustitución. }\end{array}$ & \\
\hline 37 & $\mathrm{P}$ & $\begin{array}{l}\text { Ahora, para poder saber la técnica, debemos recordar } \\
\text { la fórmula de la derivada de un producto. ¿Todos } \\
\text { recuerdan la fórmula de derivación por la regla del } \\
\text { producto? }\end{array}$ & \\
\hline 38 & estud & Si. & [Los estudiantes casi al unísono, responden ] \\
\hline 39 & $\mathrm{P}$ & ¿Cuál es? & \\
\hline 40 & estud & $\begin{array}{l}\text { Derivada de la primera por la segunda sin derivar más } \\
\text { derivada de la segunda por la primera sin derivar. A su } \\
\text { vez el profesor escribe en el tablero la fórmula: }\end{array}$ & [Todos repiten en coro] \\
\hline 41 & $\mathrm{P}$ & $u^{\prime} \cdot v+v^{\prime} \cdot u$ & $\begin{array}{l}\text { [Escribe en el tablero mientras los alumnos lo } \\
\text { dicen con palabras] }\end{array}$ \\
\hline 42 & $\mathrm{P}$ & $\begin{array}{l}\text { Ahora vamos a recordar el teorema fundamental del } \\
\text { cálculo en su } 1^{\circ} \text { parte. }\end{array}$ & $\begin{array}{l}\text { [Se acerca al escritorio del docente y mira el } \\
\text { libro y dice: ] }\end{array}$ \\
\hline 43 & $\mathrm{P}$ & ¡Mírenlo en el libro en la pág. 362! & \\
\hline 44 & $\mathrm{P}$ & $\begin{array}{l}u \text { prima por } v \text { está sumando, lo paso al otro lado a } \\
\text { restar, entonces queda la derivada el producto en } u \text { y } v \\
\text { menos la derivada de } u \text { por } v \text { sin derivar igual a } u \text { por } \\
\text { la derivada de } v \\
\text { Entonces miren: } \\
(u \cdot v)^{\prime}=u^{\prime} \cdot v+v^{\prime} \cdot u \Rightarrow(u \cdot v)^{\prime}-u^{\prime} \cdot v=u \cdot v^{\prime}\end{array}$ & \\
\hline 45 & $\mathrm{P}$ & $\begin{array}{l}\text { ¡Ahora, aplicamos el teorema fundamental del cálculo } \\
\text { es decir, recuerden que el proceso de antiderivar es el } \\
\text { inverso a la derivada! En cierto modo para eliminar } \\
\text { una de esas derivadas lo que debo hacer ¿es? ¿Qué } \\
\text { cosa? ¿Alguien sabe cómo? }\end{array}$ & $\begin{array}{l}\text { [Los estudiantes guardan silencio mientras le } \\
\text { prestan atención y toman nota. Nadie responde a } \\
\text { los interrogantes del profesor.] }\end{array}$ \\
\hline 46 & $\mathrm{P}$ & $\begin{array}{l}\text { Miren, atentos: tengo una ecuación, integramos a } \\
\text { ambos lados y me va a quedar la integral de } u . v \text { prima } \\
\text { menos } u \text { prima por } v\end{array}$ & $\begin{array}{l}\text { [Mientras el profesor hablaba escribió en el } \\
\text { tablero ] } \\
\qquad \int\left((u \cdot v)^{\prime}-u^{\prime} \cdot v\right)=\int\left(v^{\prime} \cdot u\right)\end{array}$ \\
\hline 47 & $\mathrm{P}$ & $\begin{array}{l}\text { ¡Como la integral de la izquierda es una resta, pues se } \\
\text { pude separar para calcularla! }\end{array}$ & $\begin{array}{l}\text { [Acto seguido escribe en el tablero: } \\
\qquad \int(u \cdot v)^{\prime}-\int u^{\prime} \cdot v=\int u^{\prime} \cdot v \\
Y \text { dice señalando al primera integral que resulta } \\
\text { al separar la resta] }\end{array}$ \\
\hline 48 & $\mathrm{P}$ & $\begin{array}{l}\text { ¿Qué pasa en esta primera integral ¿qué nos dice el } \\
\text { teorema fundamental del cálculo? Que cuando tengo } \\
\text { la integral de una derivada esos dos procesos se } \\
\text { anulan, Entonces ¿qué se anula aquí?, se anula esta } \\
\text { integral con esta derivada y qué queda? }\end{array}$ & $\begin{array}{l}\text { [Mientras hablaba señaló con la mano la } \\
\text { primera integral de la izquierda trazándole una } \\
\text { raya diagonal por encima al signo de la derivada } \\
y \quad \text { al signo de la integral } \\
\left.J^{\prime} u \cdot v\right)^{\prime} \text { Dice esto mientras escribe: } \\
u \cdot v-\int u^{\prime} \cdot v=\int u \cdot v^{\prime}\end{array}$ \\
\hline
\end{tabular}




\begin{tabular}{|c|c|c|c|}
\hline 49 & $\mathrm{P}$ & ¿Claro?, y ¡listo, esa es la fórmula! & \\
\hline 50 & A6 & ¡Profe eso es un poquito pesado! & \\
\hline 51 & $\mathrm{P}$ & Escribamos esta fórmula en términos de diferenciales. & \\
\hline 52 & $\mathrm{P}$ & $\begin{array}{l}\text { Eso quedaría: } u \text { por } v \text { menos } v \text {, y en vez de escribir } u \\
\text { prima escribo } d u \text {. Así nos aparece la fórmula de } \\
\text { integración por partes. }\end{array}$ & $\begin{array}{l}\text { [Dice esto mientras escribe] } \\
\qquad \int u \cdot d v=u \cdot v-\int v \cdot d u\end{array}$ \\
\hline 53 & $\mathrm{P}$ & $\begin{array}{l}\text { Esta es la regla de la integración por partes. Ahora si } \\
\text { vamos a solucionar nuestra integral que esta por acá }\end{array}$ & $\begin{array}{l}\text { [Señala la parte superior izquierda del tablero } \\
\text { donde había escrito la integral a solucionar.] }\end{array}$ \\
\hline 54 & $\mathrm{P}$ & $\begin{array}{l}\text { La fórmula de integración por partes nos dice que } \\
\text { cuando tengo la integral de una función } u \text { por la } \\
\text { derivada de una función } v \text { es igual al producto de } u \\
\text { por } v \text { menos la integral de } v \text { por } d u \text {. }\end{array}$ & $\begin{array}{l}\text { [Resalta la formula ya escrita con el marcador } \\
\text { de color rojo, encerrándola en una nube ] }\end{array}$ \\
\hline 55 & $\mathrm{P}$ & $\begin{array}{l}\text { ¿Qué tal esa fórmula no les causa alegría? ¿No los } \\
\text { emociona? }\end{array}$ & $\begin{array}{l}\text { [los estudiantes ríen mientras toman nota en sus } \\
\text { cuadernos] }\end{array}$ \\
\hline 56 & $\mathrm{P}$ & $\begin{array}{l}\text { Hay dos formas de aprenderla. ¡Una es “con la regla de } \\
\text { la vaca”! }\end{array}$ & $\begin{array}{l}\text { Dice: "Una vaca sin cola vestida de uniforme" } \\
\text { al tiempo que va señalando con la mano la u } \\
\text { para "una", la v para "vaca", el signo menos } \\
\text { para "sin", el signo de integral } \int \text { para "cola", } \\
\text { la v para "vestida" y el du para "de uniforme" } \\
\qquad u \cdot d v=u \cdot v-\int v \cdot d u\end{array}$ \\
\hline 57 & $\mathrm{P}$ & $\begin{array}{l}\text { La otra es memorizarla. ¿Complicado? ¡Esa es la } \\
\text { técnica para que la recuerden! }\end{array}$ & $\begin{array}{l}\text { [Los Estudiantes con sorpresa la repiten en } \\
\text { medio de risas.] }\end{array}$ \\
\hline 58 & A12 & $\begin{array}{l}\text { Como es profe: ¿Una vaca sin qué vestida de } \\
\text { uniforme? }\end{array}$ & $\begin{array}{l}\text { [El resto del grupo ríe al escuchar el aporte de } \\
\text { Jonathan ] }\end{array}$ \\
\hline 59 & A4 & $\begin{array}{l}\text { ¡Una vaca sin que... no! Así no dijo el profe. ¿Cómo } \\
\text { es profe? }\end{array}$ & \\
\hline 60 & $\mathrm{P}$ & Es "la regla de la vaca" & $\begin{array}{l}\text { [Y la repite a la vez que señala con la mano cada } \\
\text { letra y signo con su símil] }\end{array}$ \\
\hline 61 & A6 & ¡Chévere poder memorizarla así! & \\
\hline 62 & $\mathrm{P}$ & Volvamos al ejemplo que tenemos, ¿lo recuerdan? & $\begin{array}{l}\text { [El profesor se dirige a la parte superior } \\
\text { izquierda del tablero para mostrar el ejercicio } \\
\text { que había escrito y lo lee de la siguiente manera: } \\
\text { ] }\end{array}$ \\
\hline 63 & $\mathrm{P}$ & $\begin{array}{l}\text { Vamos a resolver la integral de equis Euler a la tres } \\
\text { equis }\end{array}$ & \\
\hline 64 & $\mathrm{P}$ & $\begin{array}{l}\text { ¿Cuáles son los indicios para saber que debo integrar } \\
\text { por partes? }\end{array}$ & \\
\hline 65 & A4 & ¡Porque hay una multiplicación entre sus términos! & \\
\hline 66 & $\mathrm{P}$ & $\begin{array}{l}\text { Muy bien. Hay una multiplicación entre dos } \\
\text { funciones no similares }\end{array}$ & \\
\hline 67 & $\mathrm{P}$ & $\begin{array}{l}\text { Acá es muy importante escoger quien ha de ser } u \\
\text { y quien } d v \text { si escojo a } u \text { esa función al derivarla debe } \\
\text { bajarle el grado, si eso no se da entonces le subo el } \\
\text { grado y significa que va mal, lo estaríamos haciendo } \\
\text { mal, vamos por mal camino }\end{array}$ & \\
\hline 68 & $\mathrm{P}$ & ¿A quién creen que debo tomar como $u$ ? & $\begin{array}{l}\text { [acto seguido sin permitir que los estudiantes } \\
\text { digan algo él menciona:] }\end{array}$ \\
\hline 69 & $\mathrm{P}$ & $u$ la tomo como $x$ y $d v$ a lo que queda & $\begin{array}{l}\text { [Mientras escribe en el tablero: ] } \\
\qquad u=x, \quad d v=e^{3 x} d x\end{array}$ \\
\hline 70 & $\mathrm{P}$ & $\begin{array}{l}\text { Entonces, pues miren lo escribo con colorcitos para } \\
\text { que no haya equívocos. La idea es que al derivar se } \\
\text { baje el grado de la integral si eso no se da entonces lo } \\
\text { hicimos mal y debemos cambiar la selección que } \\
\text { hicimos para } u \text { y para } d v \text {. }\end{array}$ & \\
\hline 71 & $\mathrm{P}$ & $\begin{array}{l}\text { ¿Que necesito para mi formula? ¡Pues determinar } \\
\text { quién es } u \text { ! }\end{array}$ & [Pero no permite que los jóvenes digan algo] \\
\hline 72 & $\mathrm{P}$ & ¡Sé quién es $u$ !, y ¿Sé quién es $d v$ ? & $\begin{array}{l}\text { [y los resalta señalándolos con la mano en el } \\
\text { tablero, pues ya los había escrito] }\end{array}$ \\
\hline 73 & $\mathrm{P}$ & Bueno miremos la fórmula & $\begin{array}{l}\text { [Y la señala en la parte superior central del } \\
\text { tablero, donde la dejo escrita] }\end{array}$ \\
\hline 74 & $\mathrm{P}$ & $\begin{array}{l}\text { Pero vean que no conozco } v \text {. Entonces ¿cómo busco } \\
v ?\end{array}$ & \\
\hline 75 & $\mathrm{~A} 12$ & ¿Integramos?, creo que es una primitiva, ¿si profe? & \\
\hline 76 & $\mathrm{P}$ & Así es, muy bien Jonathan & \\
\hline
\end{tabular}




\begin{tabular}{|c|c|c|c|}
\hline 77 & $\operatorname{Pr}$ & y no conozco $d u$ ¿cómo lo encuentro? & \\
\hline 78 & A9 & Derivamos. & \\
\hline 79 & $\mathrm{P}$ & $\begin{array}{l}\text { ¡Listo vamos hacerlo y luego reemplazamos en la } \\
\text { formula! ¿Claro para todos? }\end{array}$ & [El profesor escribe en el tablero mientras dice] \\
\hline 80 & $\mathrm{P}$ & $\begin{array}{l}\text { Aquí nos aparece } u \text { igual a equis y por tanto de } u \text { es de } \\
\text { equis. }\end{array}$ & $\begin{array}{l}\text { [En el tablero escribe: } u=x, \Rightarrow d u=d x ; y \\
\text { pregunta ] }\end{array}$ \\
\hline 81 & $\mathrm{P}$ & $\begin{array}{l}\text { ¿Y } v \text { qué es? } \\
\mathrm{dv}=\mathrm{e}^{3 \mathrm{x}} d x \quad \mathrm{v}=?\end{array}$ & [Mientras escribe:] \\
\hline 82 & $\mathrm{P}$ & A13, ¿por qué hoy está tan callado? ¿Cómo queda $v ?$ & \\
\hline 83 & A13 & Integrando profe. & \\
\hline 84 & $\mathrm{P}$ & $\begin{array}{l}\text { Si es cierto, pero ¿cómo queda } v \text { ? Es decir, ¿cuál es la } \\
\text { integral de Euler a la tres equis? }\end{array}$ & $\begin{array}{l}\text { [Dice esto, mientras escribe en el tablero: ] } \\
\int e^{3 x} d x\end{array}$ \\
\hline 85 & A9 & Da Euler a equis & \\
\hline 86 & A6 & ¡No!, ¡da un tercio Euler a la tres equis! & \\
\hline 87 & $\mathrm{P}$ & ¿Quién tiene la razón? & \\
\hline 88 & A12 & A6 profe, A9 se equivocó & \\
\hline 89 & $\mathrm{P}$ & Pase A12 al tablero y la hace. & $\begin{array}{l}\text { [Le entrega el marcador a A12 y este escribe en } \\
\text { el tablero mientras dice:] }\end{array}$ \\
\hline 90 & A12 & $\begin{array}{l}\text { Esta es una integral por sustitución simple, llamamos } u \\
\text { a tres equis, busco } d u \text { que es tres de equis. Necesito un } \\
\text { tres que multiplique al de equis luego debo multiplicar } \\
\text { y dividir toda la expresión por tres }\end{array}$ & $\begin{array}{l}{[Y \text { escribe.] }} \\
u=3 x \Rightarrow d u=3 d x\end{array}$ \\
\hline 91 & A12 & $\begin{array}{l}\text { al sustituir en la integral a términos de } u \text { me queda: un } \\
\text { tercio de la integral de Euler a la } u \text { por } d u \text { que es una } \\
\text { integral sencilla que da Euler a la } u \text {. }\end{array}$ & $\begin{array}{l}\text { [Al tiempo que hablaba y escribe en el tablero ] } \\
\qquad \int \mathrm{e}^{3 \mathrm{x}} d x=\frac{1}{3} \int e^{u} d u=\frac{1}{3} e^{3 x}+c\end{array}$ \\
\hline 92 & $\mathrm{P}$ & $\begin{array}{l}\text { A12, por el momento nos olvidamos de la constante de } \\
\text { integración c. ¿estamos? }\end{array}$ & \\
\hline 93 & $\mathrm{P}$ & $\begin{array}{l}\text { Bueno, Ya sabemos que } v \text { es un tercio de Euler a la } \\
\text { tres equis }\end{array}$ & $\begin{array}{l}\text { [ mientras escribe en el tablero ] } \\
\qquad v=\frac{1}{3} e^{3 x}\end{array}$ \\
\hline 94 & $\mathrm{P}$ & $\begin{array}{l}\text { ¿Qué más necesitamos en nuestra formula de } \\
\text { integración? Miren qué ya conocemos a } v, \text { ¿qué nos } \\
\text { haría falta? }\end{array}$ & $\begin{array}{l}\text { [Señalando la fórmula que tiene escrita en la } \\
\text { parte superior central del tablero] }\end{array}$ \\
\hline 95 & A9 & $D u$ & \\
\hline 96 & $\mathrm{P}$ & ¿Cómo hallamos $d u$ ? & \\
\hline 97 & A13 & Derivando $u$. & \\
\hline 98 & $\mathrm{P}$ & $\begin{array}{l}\text { ¿Listo!, ¡aquí sería la derivada de } u \text { que es igual a la } \\
\text { derivada de } x \text { ! }\end{array}$ & \\
\hline 99 & $\mathrm{P}$ & ¿Cuál es la derivada de $x$ ? & $\begin{array}{l}\text { [Los estudiantes al unísono dicen uno, mientras } \\
\text { el profesor escribe en el tablero] } \\
\qquad \begin{array}{r}\mathrm{u}=\mathrm{x} \quad \mathrm{dv}=\mathrm{e}^{3 \mathrm{x}} \mathrm{dx} \\
\mathrm{du}=\mathrm{dx} \quad \mathrm{v}=\frac{1}{3} \mathrm{e}^{3 \mathrm{x}}\end{array}\end{array}$ \\
\hline 100 & $\mathrm{P}$ & Uno por el diferencial que en este caso es $d x$ & [Lo señala en el escrito que hizo en el tablero ] \\
\hline 101 & $\mathrm{P}$ & ¿Listo? ¿Qué hacemos ahora? & \\
\hline 102 & estud & ¡Aplicamos la formula! & $\begin{array}{l}\text { [La mayoría de los estudiantes casi en coro } \\
\text { dicen:] }\end{array}$ \\
\hline 103 & A4 & ¿Si profe? & \\
\hline 104 & Prof. & $\begin{array}{l}\text { Así es, reemplazamos en la formula, ¿por qué duda } \\
\text { María? }\end{array}$ & $\begin{array}{l}\text { [Simultáneo el profesor escribe en el tablero] } \\
\qquad \int x e^{3 x} d x=x \cdot \frac{1}{3} e^{3 x}-\int \frac{1}{3} e^{3 x} d x\end{array}$ \\
\hline 105 & $\mathrm{P}$ & ¿Claro para todos? & \\
\hline 106 & A6 & Profe ¿podemos sacar el un tercio de la integral? & \\
\hline 107 & $\mathrm{P}$ & Y ¿por qué lo podemos hacer? & \\
\hline 108 & A12 & Porque es una constante & $\begin{array}{l}\text { [El profesor escribe en el tablero ] } \\
\qquad=\frac{1}{3} x e^{3 x}-\frac{1}{3} \int e^{3 x} d x\end{array}$ \\
\hline 109 & $\mathrm{P}$ & Esta integral ya la calculamos & $\begin{array}{l}\text { [Señala } \int e^{3 x} d x \text { y escribe la respuesta luego de } \\
\text { signo igual así: ] }\end{array}$ \\
\hline
\end{tabular}




\begin{tabular}{|c|c|c|c|}
\hline & & & $=\frac{1}{3} x e^{3 x}-\frac{1}{3} \int e^{3 x} d x=\frac{1}{3} x e^{3 x}-\frac{1}{9} e^{3 x}+c$ \\
\hline 110 & A13 & Profe ¿por qué escribió un noveno? & \\
\hline 111 & $\mathrm{P}$ & $\begin{array}{l}\text { Porque la integral de Euler a la tres equis es jun tercio } \\
\text { de Euler a las tres equis!. }\end{array}$ & $\begin{array}{l}\text { [Y señala con la mano, el lugar donde Jonathan } \\
\text { había calculado anteriormente dicha integral. } \\
\text { Los estudiantes callan, y toman nota en sus } \\
\text { cuadernos] }\end{array}$ \\
\hline 112 & $\mathrm{P}$ & $\begin{array}{l}\text { Entonces observen que pasamos de esta integral a una } \\
\text { integral más sencilla. }\end{array}$ & $\begin{array}{l}\text { [Señala con la mano la integral } \int x e^{3 x} d x \text { y luego } \\
\text { pasa la otra } \int e^{3 x} d x\end{array}$ \\
\hline 113 & $\mathrm{P}$ & $\begin{array}{l}\text { Si pasamos a una integral más sencilla partiendo de } \\
\text { una complicada, pues vamos bien, pero si pasamos una } \\
\text { integral más complicada ese no es el camino. }\end{array}$ & \\
\hline 114 & $\mathrm{P}$ & $\begin{array}{l}\text { La idea es llegar a una integral que sea más fácil de } \\
\text { resolver. }\end{array}$ & [No deja que los estudiantes respondan] \\
\hline 115 & $\mathrm{P}$ & ¿Ya terminamos? ¿Qué nos falta? & \\
\hline 116 & $\mathrm{P}$ & ¿Se podría factorizar algo? & \\
\hline 117 & A9 & Si, Euler a la tres equis & \\
\hline 118 & $\mathrm{P}$ & Muy bien, al factorizar completamente tenemos: & $\begin{array}{l}\text { [en el tablero después del ejercicio que lleva, } \\
\text { escribe un signo igual y acto seguido escribe ] } \\
\qquad \frac{1}{3} e^{3 x}\left(x-\frac{1}{3}\right)+c\end{array}$ \\
\hline 119 & $\mathrm{P}$ & ¿Claro? & $\begin{array}{l}\text { [Hay silencio mientras los estudiantes toman } \\
\text { nota. Luego de unos minutos el profesor vuelve a } \\
\text { intervenir diciendo] }\end{array}$ \\
\hline 120 & $\mathrm{P}$ & ¿Otro ejemplo o ya quedo perfecto? & \\
\hline 121 & A3 & No solo otro profe, mas... ¡el proceso es enredado! & \\
\hline 122 & $\mathrm{P}$ & $\begin{array}{l}\text { Es cuestión de práctica, en el taller } 7.1 \text { hay } 50 \\
\text { ejercicios para resolver, me deben entregar los } \\
\text { primeros } 10 \text { el viernes no lo olviden. }\end{array}$ & \\
\hline
\end{tabular}

